\title{
Comparison of vitamin D status in spring 2007 and spring 2008 among Caucasian and Asian women living in the South of England
}

\author{
P. Hadjikyriacou ${ }^{1}$, S. A. Lanham-New ${ }^{1}$, J. L. Berry ${ }^{2}$ and A. L. Darling ${ }^{1}$ \\ ${ }^{1}$ Nutritional Sciences Division, Faculty of Health and Medical Sciences, University of Surrey, Guildford, GU2 $7 H X$, \\ UK and ${ }^{2}$ Specialist Assay Laboratory (Vitamin D) and Manchester Academic Health Sciences Centre, Manchester \\ Royal Infirmary, M13 9WL, UK
}

An individual's vitamin D status depends on sunlight (UVB) exposure and dietary intake of vitamin D. 25(OH)D status throughout the year is thought to some extent to be dependent on sunlight exposure in the preceding summer. Theoretically, a cloudy and rainy summer with low temperatures could result in low vitamin D levels in the subsequent spring. Additionally, in low temperatures and in wet climates, people tend to wear heavier clothing and spend more time indoors, adding further to the reduced synthesis of vitamin D. In the summer of 2006, Southern England was less cloudy than in the summer of 2007, with a higher number of sunshine hours per day, less rainfall and a higher mean temperature. Due to the adverse weather conditions experienced in summer 2007, we hypothesised that in spring 2008 women would have lower 25(OH)D levels than they had earlier in spring 2007.

From summer 2006 to spring 2007 participants were assessed in each season for serum 25(OH)D, UVB exposure and vitamin D intake as part of the main D-FINES (Vitamin D, Food Intake, Nutrition and Exposure to Sunlight in Southern England) study. After the completion of this study in spring 2007, participants were allowed to recommence on vitamin D supplements. Subjects were re-assessed for serum $25(\mathrm{OH}) \mathrm{D}$ and UVB exposure in spring 2008 . For the current analysis, in order to exclude the impact of vitamin D supplementation on serum 25(OH)D levels, we only compared participants in spring 2007 to participants in spring 2008 who were not taking any vitamin D supplements in both seasons.

Initially, Asian and Caucasian women were analysed irrespective of menopausal status, with a paired-samples $t$-test conducted to assess differences in $25(\mathrm{OH}) \mathrm{D}$ between the two paired spring measurements. There was no significant difference in $25(\mathrm{OH}) \mathrm{D}$ between the two spring measurements (Table 1). In the Caucasian participants only, results were further analysed based on menopausal subgroups. A paired-samples $t$-test showed no significant difference in $25(\mathrm{OH}) \mathrm{D}$ between the two spring measurements in either of the menopausal subgroups. Asian subgroups were not analysed due to small subject numbers $(n=5)$.

\begin{tabular}{|c|c|c|c|c|c|c|}
\hline \multirow[b]{2}{*}{ 25(OH)D (nmol/L) } & \multicolumn{2}{|c|}{ Spring 2007} & \multicolumn{2}{|c|}{ Spring 2008} & \multirow[b]{2}{*}{$\mathrm{N}$} & \multirow{2}{*}{$\frac{\text { Paired-samples } t \text {-test }}{\mathrm{P}}$} \\
\hline & Mean & SEM & Mean & SEM & & \\
\hline Caucasian pre-menopausal & 47.59 & 3.66 & 44.97 & $4 \cdot 14$ & 18 & 0.449 \\
\hline Caucasian post-menopausal & $40 \cdot 52$ & 3.63 & 43.42 & 3.79 & 20 & $0 \cdot 392$ \\
\hline All Caucasian & 43.87 & $2 \cdot 61$ & $44 \cdot 15$ & $2 \cdot 76$ & 38 & 0.907 \\
\hline All Asian & $26 \cdot 74$ & $7 \cdot 87$ & $24 \cdot 34$ & $6 \cdot 72$ & 5 & $0 \cdot 456$ \\
\hline
\end{tabular}

The above results contradict our original hypothesis. Indeed, no significant difference was found in spring vitamin D status when preceded by a cloudy and cold summer (2007) than when preceded by a clear and warmer summer (2006). Therefore these findings suggest that a cloudy and wet UK summer may not necessarily correspond to lower vitamin D levels later on in the year. Sun exposure advice for achieving adequate vitamin D levels should therefore be simplified, with the effects of summer weather on vitamin D status not being over-emphasised in importance.

The D-FINES study was funded by the UK Food Standards Agency (Project N05064). All views are those of the authors alone. 